\title{
Are Short-Term Temporal Relationships between Depressive and Anxious Symptoms Moderated by Sex?
}

\author{
Paul E. Jose ${ }^{1 *}$, Jason S. Spendelow ${ }^{2}$, Jared Watson ${ }^{3}$ \\ ${ }^{1}$ School of Psychology, Victoria University of Wellington, Wellington, New Zealand \\ ${ }^{2}$ School of Psychology, University of Surrey, Guildford, UK \\ ${ }^{3}$ Birmingham and Solihull Mental Health NHS Foundation Trust, Birmingham, UK \\ Email: *paul.jose@vuw.ac.nz
}

How to cite this paper: Jose, P. E., Spendelow, J. S., \& Watson, J. (2020). Are ShortTerm Temporal Relationships between Depressive and Anxious Symptoms Moderated by Sex? Open Journal of Depression, 9, 58-76. https://doi.org/10.4236/ojd.2020.93006

Received: July 1, 2020

Accepted: August 9, 2020

Published: August 12, 2020

Copyright $\odot 2020$ by author(s) and Scientific Research Publishing Inc. This work is licensed under the Creative Commons Attribution International License (CC BY 4.0).

http://creativecommons.org/licenses/by/4.0/

\begin{abstract}
The chief goal of the present study was to elucidate whether the short-term temporal relationships between depressive and anxious symptoms vary by sex. Three hundred and fifty-seven undergraduate students self-reported depressive and anxious symptomatology with the Beck Depression Inventory (BDI-II) and the Beck Anxiety Index (BAI) two times 11 weeks apart. A latent variable path model analysis found support for a bi-directional relationship between depressive symptoms and anxious symptoms for the overall sample. An equality constraint analysis revealed that depressive symptoms predicted anxious symptoms over time to a similar extent as anxious symptoms predicted depressive symptoms over time. However, several temporal relationships significantly varied by sex, namely, females demonstrated greater stability of depressive symptoms over time, and evidenced a stronger cross-lag relationship from depressive symptoms at Time 1 to anxious symptoms at Time 2 , and males, on the other hand, exhibited a marginally more stable anxious symptoms test-retest relationship over time. The results supported the existence of a bi-directional relationship between depressive and anxious symptoms over a short-term period of time for emerging adults. We conclude that current states of depressive symptoms may be more influential for females' subsequent negative affective states, whereas anxious symptoms may be more important for males' subsequent negative affective states.
\end{abstract}

\section{Keywords}

Depressive Symptoms, Anxious Symptoms, Temporal Relationship, Sex

Differences, Longitudinal 


\section{Introduction}

\subsection{General Orientation}

Depression and anxiety are amongst the most prevalent of all mental health problems and represent focal points for research in various contexts around the world (Ahmen, Bowen, Feng, \& Muhajarine, 2019; Bandelow \& Michaelis, 2015; Kessler et al., 2003; Lim, Tam, Lu, Ho, Zhang, \& Ho, 2018). Investigations along conceptual and epidemiological lines have provided valuable insights but also raised new questions about the nature and prevalence of these two conditions. For instance, the degree of construct differentiation has been widely debated given high comorbidity rates (Coplan, Aaronson, Panthangi, \& Kim, 2015; Kessler et al., 1996; Ramakrishna, Cooklin, \& Leach, 2019).

The nature of the depression-anxiety relationship has been a topic of interest for decades (Cummings, Caporino, \& Kendall, 2014). Links between these two mood states have important theoretical and therapeutic implications. While commonalities exist (i.e., negative effect in the tripartite theory, see Clark \& Watson, 1991; Watson et al., 1995; Watson \& Kendall, 1989), there is evidence across disciplines that depression and anxiety are distinct phenomena (Kendall \& Brady, 1995; Kreitler, 2018). For example, aetiological differences have been found in terms of risk factors (Kessler et al., 2008): the authors found that neuroticism was a stronger predictor of generalized anxiety disorder, whereas childhood adversities more strongly predicted major depressive episodes. And Beuke, Fischer and McDowall (2003) have noted differences are found in relative effects on cognition, namely the explicit memory bias is stronger for depressed individuals, but dot-probe performance is degraded by anxiety.

Classical studies on the comorbidity of depression and anxiety (e.g., Kessler et al., 1996) have noted the co-occurrence at a single point in time of clinical diagnoses of depression (e.g., major depressive episode) and anxiety (e.g., generalized anxiety disorder). However, in order to understand comorbidity better, studies examining transitions between diagnoses are needed. In this vein, scholarly work on the comorbidity of depression-anxiety early in the life-course has been conducted within a developmental psychopathology framework. The prevailing view here is that a uni-directional relationship exists in that anxiety temporally precedes depression in the life-course (e.g., Chaplin, Gillham, \& Seligman, 2009; Starr, Stroud, \& Li, 2016; Wittchen, Kessler, Pfister, \& Lieb, 2000). These findings are based on the earliest reports of mood disorders and reveal information about incipient directions of types of negative mood in childhood and adolescence.

Does this temporal anxiety to depression relationship apply later in the life-span, and does it describe short-term relationships, e.g., associations over months? Studies with adults suggest that depressive and anxious symptoms likely share a more balanced bi-directional relationship over years or months. Indeed, a relationship of this nature has been reported in several studies (e.g., Het- 
tema, Prescott, \& Kendler, 2003; Kessler et al., 2002), and epidemiological data suggests the presence of one mood state represents a risk factor for the other (Cummings et al., 2014; Moffitt et al., 2007; Skouteris, Wertheim, Rallis, Milgrom, \& Paxton, 2009).

Most of the research on this topic has employed either adults or adolescents as participants, so it is important to document whether this phenomenon also applies to the intervening life-span period of emerging adults. However, little research has examined associations between depressive symptoms and anxious symptoms longitudinally with emerging adults. One exception is a large-scale study by Lovibond (1998), in which the Depression Anxiety Stress Scale (DASS) was administered to a group of 3540 university students over a time period from 1986 to 1991. The DASS, which yields continuous estimates of depressive and anxious symptoms, was again administered in 1994 to 882 members of their original sample to produce a time 2 sample. These researchers found support for a small bidirectional relationship between anxious symptoms and depressive symptoms. In addition to the large sample size, this study was also noteworthy for its use of structural equation modelling (SEM), which is appropriate for large samples which yield continuous-type data. Latent variable SEM has advantages over other methods because relationships can be measured without error and rigorous evaluation of simultaneous residualised effects can also be identified (Little, 2013).

The present study used a similar approach, namely using continuous variable assessments of depressive and anxious symptoms in a longitudinal study with university students. Our study investigated the presumed bi-directional relationship over a shorter amount of time than Lovibond did: in our case, 11 weeks. We would argue that using an emerging adult sample to collect longitudinal data is important because it is likely to illuminate important relationships between depressive and anxious symptomatology at a period in their lives when life-long patterns of mood regulation are being consolidated (Wood et al., 2018). In the first instance, we expected that the data would confirm the presumed bi-directional relationship between depressive and anxious symptoms over time for the overall sample. And second, we sought to obtain novel information about whether this presumed bi-directional relationship might vary by sex, shedding light on an important moderator of this age group.

\subsection{Gender Differences in Relationships between Depressive Symptoms and Anxious Symptoms}

Sex represents a key variable of interest when conducting research on depressive symptoms and anxious symptoms. Robust gender differences in the trajectories and prevalence of internalising problems is one reason for this importance. Rates of depressive symptoms begin to diverge in early adolescence and, by mid to late adolescence, females exhibit rates up to twice those found for males (Hankin, 2009; Nolen-Hoeksema \& Jackson, 2001; Salk, Hyde, \& Abramson, 
2017). This ratio holds true across much of the lifespan (Albert, 2015). Females also report higher levels of anxious symptoms (Jalnapurkar, Allen, \& Pigott, 2018) with divergence in certain symptoms in early adolescence (Hale, Raaijmakers, Muris, van Hoof, \& Meeus, 2008). Rates of generalised anxiety disorder for females are approximately three times that of males (Bandelow \& Michaelis, 2015). In line with these findings, females return higher scores on psychometric instruments such as the Beck Depression Inventory-II (BDI-II) (Beck \& Steer, 1993; de Sa Jr. et al., 2019) and Beck Anxiety Inventory-II (BAI-II) (Bardhoshi, Duncan, \& Erford, 2016; Beck, Steer, \& Brown, 1996). Based on these findings, we expected to find our female participants reporting higher levels of both depressive and anxious symptoms.

Beyond mean group differences, we are arguably more interested in examining how sex might moderate relationships between variables. Given the relevance of gender differences for mood disorders, it is perhaps surprising that this variable has received limited attention as a moderator of the temporal relationship between depressive symptoms and anxious symptoms. One exception comes from the work of Chaplin, Gillham and Seligman (2009). Using the Child Depression Inventory (CDI) and the Revised Children's Manifest Anxiety Scale (RCMAS), these authors measured depression symptoms and three forms of anxious symptoms (worry and oversensitivity; social concerns and concentration; and physiological anxiety) in a sample $(\mathrm{N}=113)$ of 11 - 14-year-olds at two time points separated by a 12 -month interval. Regression analysis revealed that worry and oversensitivity predicted subsequent depressive symptoms more strongly for females than males. In contrast, a second study, by Parker and Hadzi-Pavlovic (2004), analysed data from the National Comorbidity Survey $(\mathrm{N}=5877)$ and found that the onset of anxiety (as measured by the Composite International Diagnostic Interview, CIDI) in adolescence/early adulthood increased the risk for subsequent depressive symptoms more so for males than females. Since neither of these studies focused on an emerging adult sample, and the two findings are inconsistent, new work is needed to document conclusive patterns of influence between depressive and anxious symptoms in emerging adulthood.

\subsection{Predictions}

In the present study, the Beck Depression Inventory-II (BDI-II) and the Beck Anxiety Index (BAI) were administered to emerging adults attending university at two points in time separated by 11 weeks in order to investigate the short-term temporal relationships between depressive symptoms and anxious symptoms. The Beck inventories are widely used self-report measures of depressive symptoms and anxious symptoms (Whisman \& Richardson, 2015) and were deemed appropriate in the present context as they capture the continuous levels of both depressive symptoms and anxious symptoms well, while also minimising construct overlap. 
The first prediction was that, compared to males, females would report higher rates of depressive symptoms and anxious symptoms at both time periods (Hypothesis 1), consistent with previous research (e.g., Albert, 2015).

The second prediction was that a bi-directional relationship would be found between depressive symptoms and anxious symptoms scores over time for the whole sample (Hypothesis 2). This prediction was based on previous studies (e.g., Hettema et al., 2003; Kessler et al., 2002; Moffitt et al., 2007; Starr et al., 2016), although none of these studies examined with issue with this type of sample nor for this span of time.

The third prediction was that we expected sex to moderate these relationships. Specifically, we predicted that anxious symptoms would predict subsequent depressive symptoms more strongly for males (compared with females) based on the large sample study by Parker and Hadzi-Pavlovic (2004) (Hypothesis 3). In contrast, we expected that depressive symptoms would be more strongly predictive of subsequent anxious symptoms for females than for males. This prediction was based on the greater tendency for females to exacerbate negative affect: Johnson and Whisman's (2013) meta-analysis shows that females ruminate more than males; and Rose (2002) has documented that females co-ruminate with friends more than do males.

\section{Method}

\subsection{Participants}

Participants were first-year university students enrolled in one of three typical first year courses: Politics, Computer Science, and Marketing. No exclusionary criteria were used, thus, all enrolled students who attended class on these days were included in the study. Five hundred and fifty-six participants completed the questionnaire packet at time 1, and 357 participants completed it a second time at time 2. Data analyses were performed on the data provided by participants who completed the surveys at both time points. Attrition was due to students withdrawing from the class or being absent on the second time of measurement. An attrition analysis showed that students tested at both time points reported about equal levels of anxious symptoms but lower levels of depressive symptoms than students who were not retained $(F(1,555)=8.98, p=.001)$, but the effect size was small $\left(\eta^{2}=.01\right)$. Fifty-seven percent of the final sample was male and forty-three percent was female.

The mean age of the sample at Time 1 was 20.21 years $(S D=4.92)$. Eighty-four percent of the sample reported their age between 17 and 21 years, and the rest fell in the range from emerging adulthood to middle age adults (maximum $=56$ years). Sixty-two percent of the total sample self-identified as European New Zealand, $9.8 \%$ as Chinese, $5.2 \%$ as European, $4.9 \%$ as Maori, $2.5 \%$ as Indian, $1.4 \%$ as Samoan, $4.3 \%$ as "Other-Asian", and $6.4 \%$ chose not to specify their ethnicity. Maori and Pacific Island students were under-represented and Asian and Western international students were slightly over-represented in this 
New Zealand sample.

\subsection{Measures}

The test packet included the Beck Depression Inventory-2nd edition (BDI-II; Beck, Steer, \& Brown, 1996) and the Beck Anxiety Inventory (BAI; Beck \& Steer, 1993). A range of demographic information was also collected: age, sex, and self-identified ethnicity. In one half of the test packets, the BDI-II was administered first, and in the other half the BAI was administered first. A non-significant order effect was found in a MANOVA, $p<.20$.

The Beck Depression Inventory-Second Edition (Beck, Steer, \& Brown, 1996). The BDI-II was developed to assess "the severity of depression in diagnosed patients", and to detect "possible depression in normal populations". The BDI-II is made up of 21 items and each item consists of 4 self-report statements representing different levels of severity for each particular symptom. The four-point scale is scored from 0 to 3 . A respondent can therefore obtain a score between 0 and 63 .

Studies examining the validity and reliability of the BDI-II have shown that it is internally consistent with coefficient alphas typically falling above .85 (Whisman \& Richardson, 2015). The scale has also demonstrated acceptable convergent validity. The BDI-II is highly correlated with the BDI, i.e., $r=.93$ (Beck et al., 1996), and with a range of other measures of depressive symptoms such as the Beck Hopelessness scale, $r=.68$, and the Revised Hamilton Psychiatric Rating Scale for Depression, $r=.71$.

The BDI-II has demonstrated acceptable discriminant validity from anxious symptoms with correlations of .60 and .56 with the BAI (Beck et al., 1996; Osman et al., 1997). Osman et al. (1997) also observed the BDI-II to be only moderately correlated with the anxiety subscale of the DASS, $r=.44$, and the anxious arousal subscale of the MASQ at .46. The BDI in the present study yielded Cronbach's alphas of .89 and .91 at the two times of measurement.

The Beck Anxiety Inventory (BAI; Beck \& Steer, 1993). Anxious symptoms were measured with the Beck Anxiety Inventory. The BAI was developed to address deficiencies with existing measures of anxiety, specifically the failure to reliably discriminate anxious symptoms from depressive symptoms. Like the BDI, the BAI was not designed as a diagnostic instrument but to assess overall levels of anxious symptoms. Each of the 21 items describes a single symptom of anxiety and respondents are requested to rate their response on a four-point scale with 0 representing "not at all" and 3 representing "severely, I could barely stand it". Therefore, a total score on the BAI can range from 0 to 63 , which is an identical range as the BDI-II.

The BAI has very good internal consistency, i.e., typically falling above .90 (Bardhoshi et al., 2016). The BAI has exhibited moderate correlations with other clinician-based and self-report measures of anxious symptoms. For instance, the BAI was found to be correlated with the Hospital Anxiety Rating 
Scale by .51 and .56 and with the STAI trait and state at between .60 and .50 (Julian, 2011).

The BAI has similar discriminant validity to the BDI-II. Frydrich, Dowdall and Chambless (1992) reported that the BAI was correlated with the BDI at .50. Beck, Steer and Carbin (1988) also reported a low discriminant correlation of .28 for the BAI with the Hamilton Depression Rating Scale. The BAI in the present study yielded Cronbach's alphas of .90 and .91 at the two times of measurement.

\subsection{Procedure}

Students completed the packet of questionnaires, consisting of the BDI-II and the BAI, at two time points separated by a period of eleven weeks. The first administration took place in week one of the first terms of the school year, and the second administration took place in the final week of the first term. The questionnaire packets were completed by students in university lecture theatres during class time. The questionnaires were administered at the beginning of each class session. The author explained to the students that he was conducting research on depressive symptoms and anxious symptoms in students. Students were asked to read and sign the consent form and if they did so, then to complete the questionnaire packet. Ethical approval for the project was obtained from the School of Psychology Ethics Committee. At the end of the second administration, students were also provided with a detailed explanation of the purposes and aims of the research. Students were provided with contact details for the Student Counselling Service and encouraged to seek psychological help if they were feeling depressed or anxious.

\section{Results}

\subsection{Descriptive Statistics and Preliminary Analyses}

Means, standard deviations, and correlations for observed scores on the BDI-II and BAI provided by participants who yielded data at both Times 1 and 2 are presented in Table 1. As expected, depressive and anxious symptoms were significantly associated concurrently as well as longitudinally: Table 1 reports both types of associations fell in the moderate range. Also, the Cronbach's alphas of the two measures at both time points were excellent. A visual inspection of the distribution of scores suggested that the distributions of scores in the present data set were positively skewed and possibly kurtotic as well. This type of distribution, however, was expected given the nature of the measures for a non-clinical sample. Significance tests recommended by Tabachnick and Fidell (2019) indicated that all four distributions were significantly positively skewed and the presence of moderate kurtosis was found as well. Tabachnick and Fidell have recommended applying a square-root transformation in this situation, so it was employed and both skewness and kurtosis were attenuated. Analyses were 
Table 1. Correlations and descriptive statistics for the entire sample at both time points.

\begin{tabular}{ccccc}
\hline & BDI Time 1 & BAI Time 1 & BDI Time 2 & BAI Time 2 \\
\hline BDI Time 1 & & .589 & .642 & .454 \\
BAI Time 1 & & & .478 & .568 \\
BDI Time 2 & .89 & .90 & .91 & .614 \\
Cronbach's alpha & 9.31 & 9.70 & 9.12 & .91 \\
Mean & 7.72 & 8.50 & 7.80 & 8.46 \\
\multicolumn{1}{c}{$S D$} & & & & 8.19 \\
\hline
\end{tabular}

Note: $\mathrm{N}=359 . \mathrm{SD}=$ standard deviation; BDI = Beck Depression Inventory; BAI = Beck Anxiety Inventory.

performed on these transformed data, but untransformed data are reported in Table 1.

\subsection{Mean Group Differences across Time}

A repeated measures MANOVA was computed to determine whether depressive symptom and/or anxious symptom scores changed over the 11 weeks of the study. Sex was included as a fixed factor to see whether change over time was moderated by sex. A multivariate main effect for time was found, Wilk's $\lambda$ $=.974, F(2,355)=4.63, p<.01$, partial $\eta^{2}=.03$, but the only significant univariate effect was for anxious symptoms, $F(1,355)=9.28, p<.01$, partial $\eta^{2}=.03$. Table 1 shows that anxious symptoms scores decreased modestly from Time 1 to Time 2.

No time $\mathrm{X}$ sex interaction was found, which suggests that stability of depressive symptoms over time and the decrease in anxious symptoms occurred similarly for both males and females. However, and as expected, a significant sex main effect was obtained for both depressive symptoms and anxious symptoms, $F \mathrm{~s}(1,355)=11.32$ and $13.22, p s<.001$, partial $\eta^{2} s=.03$ and .04 . In both cases, females $(M s=10.52$ and 10.56$)$ reported higher levels of depressive symptoms and anxious symptoms respectively than males $(M \mathrm{~s}=8.07$ and 7.75$)$, consistent with Hypothesis 1.

\subsection{Latent Variable Longitudinal Path Analyses}

The second hypothesis of the present study was that a bidirectional relationship would be found between depressive symptoms and anxious symptoms over time. In order to assess this temporal relationship most sensitively, we elected to use latent variable longitudinal cross-lag path analysis with parcels. Little, Cunningham, Shahar and Widaman (2002) have noted that using three parcels for each latent construct is mathematically optimal. Since internal reliabilities of these two measures were found to be high in the present study (i.e., 89 to .91 for depressive symptoms and .90 to .91 for anxious symptoms), we felt that it was justified to use parcels to simplify the structural equation model. Parcels were constructed by adding every fourth item into each parcel (e.g., BDI parcel 1 contained BDI1, BDI4, BDI7, and so forth), and then dividing by the number of 
items. The AMOS (Arbuckle, 2014) structural equation modelling platform was used to estimate the resulting path model (see Figure 1). Although not displayed, the model included estimates of autocorrelated error (i.e., covariances between identical parcels across time).

The present study used several indices of absolute fit ( $\chi^{2} / \mathrm{df}$, RMSEA, and critical N), and several indices of incremental fit (CFI, NFI, RFI, and TLI). All of the obtained fit indices for the three models (see Table 2) fell within the good fitting range.

Table 2. Model fit indices for the SEM models.

\begin{tabular}{cccc}
\hline Model fit indices & Total sample & Males & Females \\
\hline Chi-square & 101.7 & 69.7 & 104.6 \\
$d f$ & 42 & 42 & 42 \\
$\chi^{2} /$ df ratio & 2.42 & 1.66 & 2.49 \\
RMSEA & .04 & .04 & .07 \\
NFI & .98 & .97 & .96 \\
RFI & .96 & .95 & .92 \\
CFI & .99 & .99 & .97 \\
TLI & .98 & .98 & .95 \\
Critical N & 413 & 381 & 189 \\
\hline
\end{tabular}

Note: $\mathrm{df}=$ degrees of freedom; RMSEA $=$ root mean square error of approximation; NFI, RFI, CFI, and Critical $\mathrm{N}$ are model fit indices.

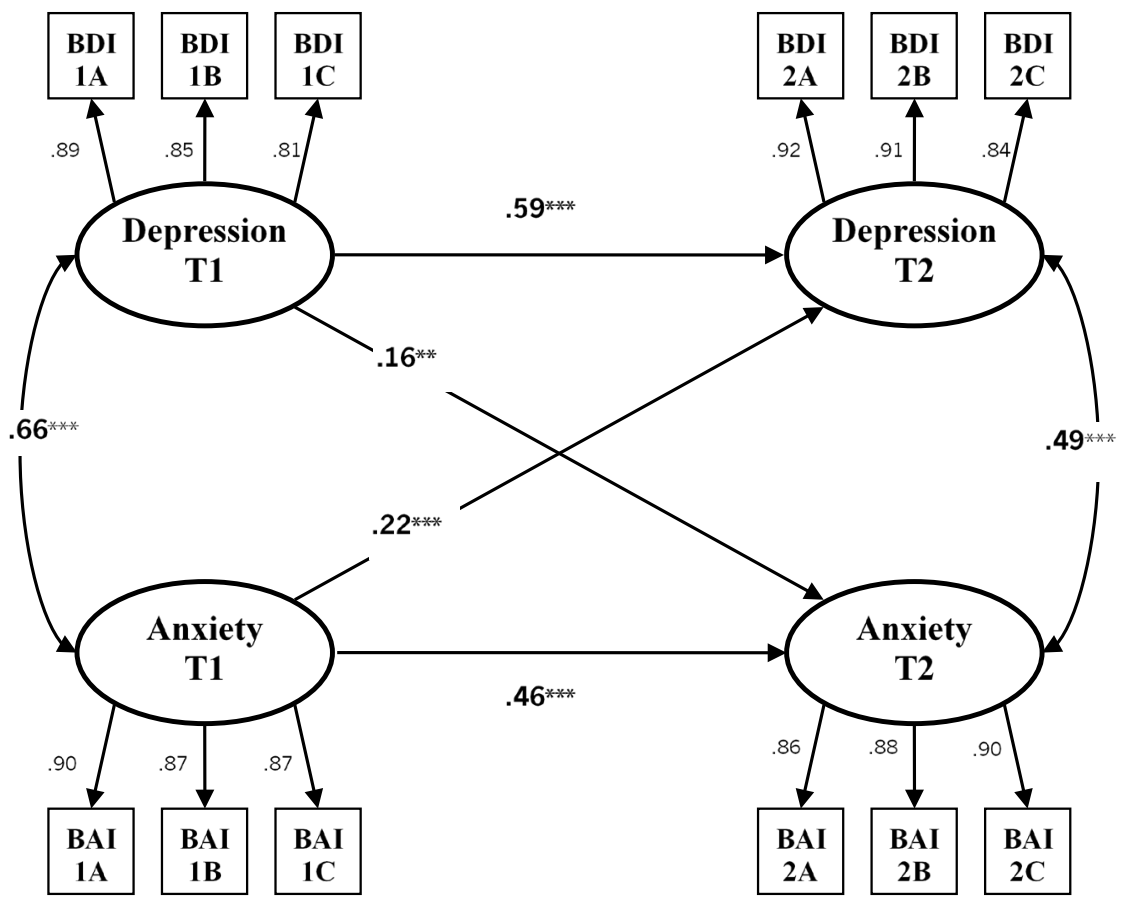

Figure 1. Obtained estimates for the longitudinal cross-lag path model for the entire sample. Note: Although not shown for the sake of brevity, autocorrelated error terms between time points and residual error terms for the endogenous variables were included in the SEM model. ${ }^{* *} p<.01 ;{ }^{* * *} p<.001$. 
Next the parameter estimates in this model were examined. The factor loadings for each indicator (see Figure 1) were uniformly large and statistically significant, and this result suggested that the item parcels for the BDI and BAI scales well represented the latent variables of depressive symptoms and anxious symptoms. As expected, the stability path coefficients for depressive symptoms and anxious symptoms over time were both moderate in size. The beta weight for the stability path from BDI Time 1 to BDI Time 2 was .59 and was statistically significant $(p<.001)$, and the beta weight from BAI Time 1 to BAI Time 2 was .46 and was also significant $(p<.001)$. These results suggested that anxious symptoms and depressive symptoms scores were moderately stable over the eleven-week period between times of measurement.

The cross-lag coefficients in this model can be seen in Figure 1 and, and as predicted, statistically significant estimated cross-lag paths were obtained between Depressive symptoms Time 1 on Anxious symptoms Time 2 as well as between Anxious symptoms Time 1 on Depressive symptoms Time 2. These results are consistent with Hypothesis 2 that a bidirectional relationship between the symptoms of depression and anxious symptoms over time would be obtained.

\section{Testing Strengths: Equality Constraints}

Two comparisons of strength of estimated parameters were performed. First, we sought to determine whether the two cross-lag paths were equivalent in strength in Figure 1: namely, whether the depressive symptoms to anxious symptoms link $(\beta=.22)$ was stronger than the anxious symptoms to depressive symptoms $(\beta=.16)$ link. The first equality constraint test revealed that the chi-square change value was .10 with $1 \mathrm{df}, p>.50$, clearly a non-significant result. We therefore concluded that the two cross-lag paths were about equivalent in strength. The second test compared the stabilities of depressive symptoms and anxious symptoms. The chi-square change value in this case was 4.50 with $1 \mathrm{df}$, $p<.05$. This result permits us to conclude that depressive symptoms $(\beta=.59)$ was statistically more stable over time than anxious symptoms $(\beta=.46)$.

\subsection{Sex Differences in the Cross-Lag Path Model}

To determine whether males and females differed or not in the expression of the obtained overall bi-directional process, we performed multi-group analyses with equality constraints. The first step was to run a baseline model with the two groups specified. An examination of the resulting path models (see Figure 2) shows that we obtained some interesting potential differences between the two groups.

The second step was to test configural invariance by holding all four temporal paths equal (Cheung \& Rensvold, 2002), and we observed a significant deterioration in the chi-square value $(p<.05)$. Subsequent probes of the two stabilities and the two cross-lag paths yielded the following two significant results: 1) female participants exhibited more stable depressive symptoms scores than males 


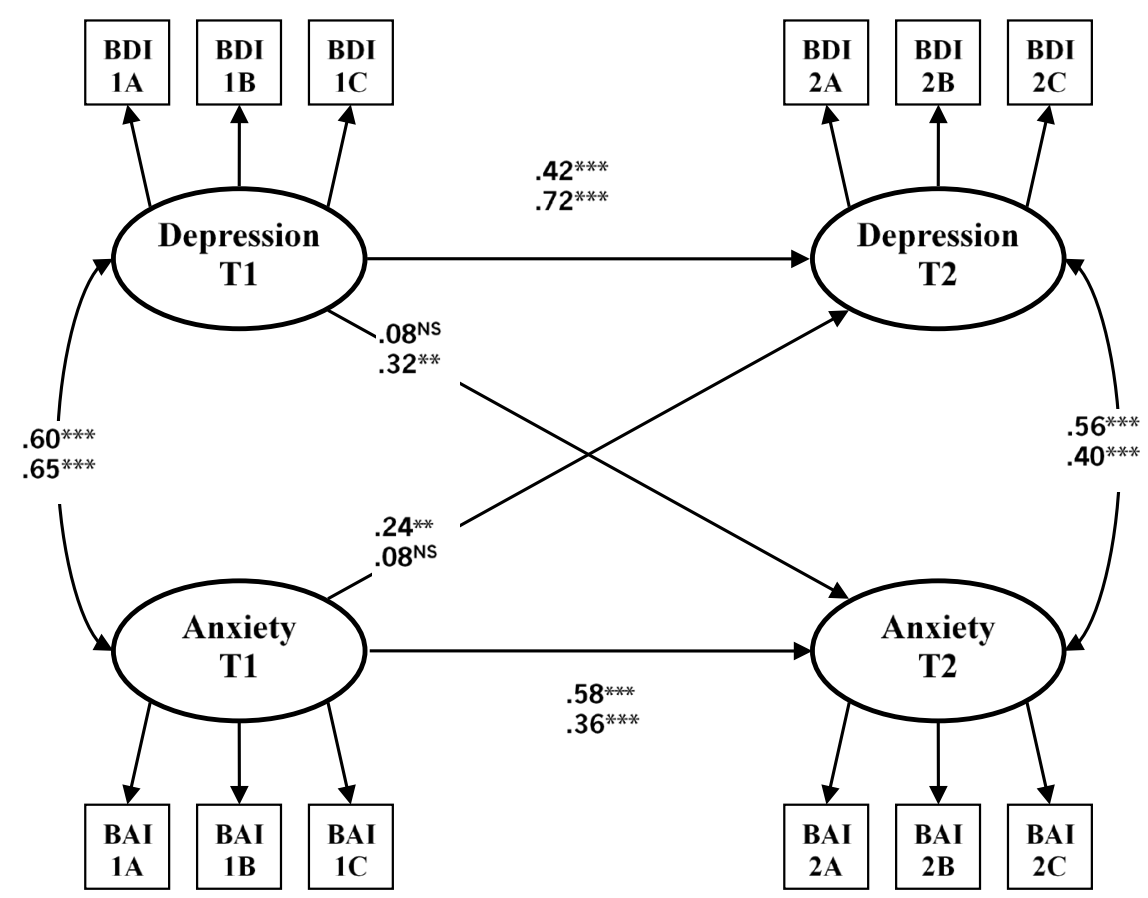

Figure 2. Obtained estimates for the longitudinal cross-lag path model for males and females. Note: Coefficients on top refer to the male sample and coefficients on the bottom refer to the female sample. Although not shown for the sake of brevity, autocorrelated error terms between time points and residual error terms for the endogenous variables were included in the SEM model. NS = non-significant; ${ }^{* *} p<.01 ;{ }^{* *} p<.001$.

( $\beta s=.72$ vs. .42 respectively, chi-square change $=6.6, p<.01)$; and 2$)$ females exhibited a stronger depressive symptoms to anxious symptoms cross-lag path than males ( $\beta s=.32$ vs. .08 respectively, chi-square change $=4.0, p<.05$ ). In addition, we obtained a marginally significant result for the stability of anxious symptoms: males demonstrated somewhat greater stability than females ( $\beta \mathrm{s}$ $=.58$ vs. .36 respectively, chi-square change $=2.9, p=.08$ ). No significant sex difference was noted for the anxious symptoms to depressive symptoms cross-lag path. Taken together these results suggest that the depressive symptoms variable was more influential for females' subsequent affective states, whereas the anxious symptoms variable was more important for males' affective states, consistent with Hypothesis 3.

\section{Discussion}

The current study was designed to add to our limited understanding of the temporal link between depressive symptoms and anxious symptoms, particularly with regard to potential sex differences in this relationship among emerging adults. The first prediction concerned mean group differences, namely, that, compared to males, females would report higher rates of depressive and anxious symptoms at both time periods (T1 and T2). Hypothesis 1 was supported by empirical findings which showed that females reported higher scores for both anxious and depressive symptoms, consistent with a large body of previous work 
identifying higher rates of depressive symptoms and anxious symptoms amongst females (e.g., Albert, 2015; Jalnapurkar et al., 2018).

Our second prediction was that a bi-directional relationship would be found between depressive and anxious symptoms scores for the overall sample. This hypothesis was confirmed by the present data and is supported by previous work in this area (e.g., Hettema et al., 2003; Kessler et al., 2002; Lovibond, 1998; Moffitt et al., 2007). Our results from the present short-term longitudinal study with an emerging adults sample suggest that depressive and anxious symptoms predict increases in the other mood state through time, and to about the same extent. The current study is perhaps the only study in the literature that has involved a short-term longitudinal period of time and relied on data obtained from emerging adults. This finding suggests that the comorbid relationship between depressive and anxious symptoms is self-reinforcing, and further, that these influences are about of equal strength.

The final prediction for this study was that these relationships would be moderated by sex over time. Contrary to predictions, results indicated that depressive symptoms scores were more stable for females than for males. To support this unanticipated finding, the literature (e.g., Essau, Lewinsohn, Seeley, \& Sasagawa, 2010) has noted that females evidence longer depressive episodes, and although our time frame is relatively short, our finding is consistent with this general trend.

The third prediction specifically suggested that anxious symptoms would more strongly predict depressive symptoms over time for males than females. Figure 2 shows that the opposite finding was obtained: females exhibited a stronger cross-lag path than did males. And last, a marginally significant finding showed that males' anxious symptoms scores were somewhat more stable than females' scores. Taken together, these findings suggest that females' negative mood states are more constrained by their levels of relatively higher and more stable depressive symptoms, and, in contrast, there is a suggestion that males' negative mood states are more constrained by levels of their relatively more stable, albeit lower, anxious symptoms. The current study findings for males are inconsistent with the work of Parker and Hadzi-Pavlovic (2004) whose study focused on adulthood, but are more consistent with those of Chaplin et al. (2009) whose study focused on adolescence. At the same time, our study differed from both of these other studies in terms of depression and anxiety assessment, length of time between times of measurement, and sample selection, so it is difficult to know how our study's finding concurs with Chaplin et al.'s study rather than Parker and Hazi-Pavlovic's. Further, since we did not obtain biological markers of age (e.g., puberty, secondary sex characteristics, hormones, etc.), we cannot provide enlightenment about why we obtained the findings that we did. Additional research is needed to determine how emerging adults evidence patterns of association over time more similar to adolescents or to adults, and we would suggest the inclusion of both biological (e.g., hormonal levels) and psychological (e.g., emotional maturity) markers to disambiguate these complex re- 
lationships.

\subsection{Implications of Current Findings}

Two major implications of our findings deserve note. First, the bi-directional influences of depressive symptoms and anxious symptoms on each other over time suggest, as noted by others (Lovibond, 1998), that a diagnosis of one negative mood state implies that the individual is at risk for developing the other negative mood state. Clinicians would do well to screen for both negative mood states at the same time, and to monitor levels of symptoms of both over time. Our findings also suggest that, left unchecked, the interrelated influences of these mood states are likely to create a "downward spiral" of mood and affect (Hubley \& Dimidjian, 2017). It is critical to bring ameliorating impacts to the individual, e.g., counselling, therapy, or positive mood interventions (Garland, Fredrickson, Kring, Johnson, Meyer, \& Penn, 2010), and at the same time be cognizant of the potential linkages between depressive and anxious symptoms. For example, will a young person's sense of loss or lack of control, which often undergirds a depressed outlook on life, also feed a sense of impending doom typical of anxiety?

The second major implication is that the bi-directional relationship between depressive symptoms and anxious symptoms may differ in significant ways between males and females. For example, the data seem to suggest that levels of depressive symptoms are higher and more stable for females than males, whereas anxious symptoms levels seem to change more for females. If this finding is replicated for this age group, it would have significant repercussions for clinicians attempting to tackle mood disorders in college and university young people. Tackling depressive symptoms in young women and targeting anxious symptoms in young men might trigger more enduring and pervasive change in psychological adaptation than the other way around. However, these results would need to be replicated in other samples before whole-scale changes in therapeutic approaches are recommended.

\subsection{Limitations and Future Directions}

First, the use of a non-clinical sample in the current study raises the issue of applicability of these findings to clinical populations. The "continuity of dysfunction" debate, identified by Flett, Vredenburg, and Krames (1997), contrasts the view that the distinction between sub-clinical and clinical symptoms is qualitative with those who argue that the difference is quantitative. While arguments exist in support of both continuity (Vredenburg, Flett, \& Krames, 1993) and discontinuity (Coyne, 1994), the former is accompanied by significant empirical support (e.g., Lovibond, 1998) and Kessler (2007) also points out that those individuals identified at the mild end of symptom severity are still at risk of demonstrating outcomes indicative of mental disorder over time. Researchers should replicate this identified bi-directional relationship with other non-clinical and community samples, as well as attempt to extend the finding to clinical 
samples to determine its generalizability.

A second limitation was the use of junior undergraduate students, who were tested early and late in a given trimester. Fluctuations of anxious and depressive symptoms occur over the course of an academic term for individuals operating in this context, so future work should capture these mood assessments at different times to verify that contextual pressures do not significantly alter the bi-directional effect demonstrated here. Further, not all emerging adults attend university, so this sample was not representative of all emerging adults.

Third, attrition of more depressed individuals in our longitudinal study suggests that we obtained differential attrition. Nevertheless the size of this effect was small, and likely only slightly affected the obtained results.

Fourth, the temporal relationship in the present study was 11 weeks, and future work should investigate both shorter and longer periods of time to see whether bi-directionality is found at all time lengths. Fifth and last, the obtained sex difference in the present study is intriguing and points toward needed future work, but our present study was limited in that it was not designed to explicate mechanisms of obtained sex differences. Future work should identify what psychological, sociological, genetic, hormonal, and physiological differences between males and females are responsible for this observed sex difference (see Hutt, 1978). Findings from these multi-disciplinary threads of research would prove useful for the design and implementation of sex-tailored screening tools and interventions.

\subsection{Conclusions}

The present investigation into the temporal relationships between depressive symptoms and anxious symptoms obtained three key findings:

- females reported higher levels of both negative mood states than males;

- depressive symptoms predicted increases in anxious symptoms over 11 weeks' time at about the same rate as anxious symptoms predicted increases in depressive symptoms over time, evidencing a bi-directional temporal relationship; and

- females exhibited greater stability of depressive symptoms over time than males, females evidenced a stronger cross-lag prediction from depressive symptoms to anxious symptoms than males, and males evinced marginally more stable levels of anxious symptoms than females.

Taken together we conclude from these findings that although the overall sample demonstrated that depressive symptoms predicted anxious symptoms about as strongly as anxious symptoms predicted depressive symptoms over time, several notable sex differences in the dynamics of these two negative mood states were found in this sample of emerging adults. The chief implications are that researchers and clinicians need to be aware of the general bi-directional relationship over time identified here between these two co-morbid mood disorders, as well that sex differences exist in this bi-directional pattern. In particular, 
females' trajectories of negative mood states seem to emanate from baseline depressive states more so than from anxious states. Further work is needed to characterise more accurately how various moderators (e.g., family psychiatric history) of the basic bi-directional relationship between depressive and anxious symptoms plays out over different lengths of time and in different contexts.

\section{Acknowledgements}

Appreciation is expressed to the participants of the study and to the instructors who permitted data collection in their classes. Reprints can be obtained by writing to the first author at: School of Psychology, P.O. Box 600, Victoria University of Wellington, Wellington, New Zealand; paul.jose@vuw.ac.nz (e-mail); or +64-04-463-5402 (fax).

\section{Conflicts of Interest}

The authors declare no conflicts of interest regarding the publication of this paper.

\section{References}

Ahmen, A., Bowen, A., Feng, C. X., \& Muhajarine, N. (2019). Trajectories of Maternal Depressive and Anxiety Symptoms from Pregnancy to Five Years Postpartum and Their Prenatal Predictors. BMC Pregnancy and Childbirth, 19, Article No. 26. https://doi.org/10.1186/s12884-019-2177-y

Albert, P. R. (2015). Why Is Depression More Prevalent in Women? Journal of Psychiatry and Neuroscience, 40, 219-221. https://doi.org/10.1503/jpn.150205

Arbuckle, J. L. (2014). Amos (Version 23.0) [Computer Program]. Chicago, IL: IBM SPSS.

Bandelow, B., \& Michaelis, S. (2015). Epidemiology of Anxiety Disorders in the 21st Century. Dialogues in Clinical Neuroscience, 17, 327-335.

Bardhoshi, G., Duncan, K., \& Erford, B. T. (2016). Psychometric Meta-Analysis of the English Version of the Beck Anxiety Inventory. Journal of Counseling and Development, 94, 356-373. https://doi.org/10.1002/jcad.12090

Beck, A. T., \& Steer, R. A. (1993). Beck Anxiety Inventory manual. San Antonio, TX: Psychological Corporation.

Beck, A. T., Steer, R. A., \& Brown, G. K. (1996). Beck Depression Inventory-II. San Antonio, TX: Psychological Corporation. https://doi.org/10.1037/t00742-000

Beck, A. T., Steer, R. A., \& Carbin, M. G. (1988). Psychometric Properties of the Beck Depression Inventory: Twenty-Five Years of Evaluation. Clinical Psychology Review, 8 , 77-100. https://doi.org/10.1016/0272-7358(88)90050-5

Beuke, C. J., Fischer, R., \& McDowall, J. (2003). Anxiety and Depression: Why and How to Measure Their Separate Effects. Clinical Psychology Review, 23, 831-848. https://doi.org/10.1016/S0272-7358(03)00074-6

Chaplin, T. M., Gillham, J. E., \& Seligman, M. E. P. (2009). Gender, Anxiety, and Depressive Symptoms: A Longitudinal Study of Early Adolescents. Journal of Early Adolescence, 29, 307-327. https://doi.org/10.1177/0272431608320125

Cheung, G. W., \& Rensvold, R. B. (2002). Evaluating Goodness-of-Fit Indexes for Testing 
Measurement Invariance. Structural Equation Modeling, 9, 233-255. https://doi.org/10.1207/S15328007SEM0902_5

Clark, L. A., \& Watson, D. (1991). Tripartite Model of Anxiety and Depression: Psychometric Evidence and Taxonomic Implications. Journal of Abnormal Psychology, 100, 316-336. https://doi.org/10.1037/0021-843X.100.3.316

Coplan, J. D., Aaronson, C. J., Panthangi, V., \& Kim, Y. (2015). Treating Comorbid Anxiety and Depression: Psychosocial and Pharmacological Approaches. World Journal of Psychiatry, 5, 366-378. https://doi.org/10.5498/wjp.v5.i4.366

Coyne, J. C. (1994). Self-Reported Distress: Analog or Ersatz Depression? Psychological Bulletin, 116, 29-45. https://doi.org/10.1037/0033-2909.116.1.29

Cummings, C. M., Caporino, N. E., \& Kendall, P. C. (2014). Comorbidity of Anxiety and Depression in Children and Adolescents: 20 Years after. Psychological Bulletin, 140, 816-845. https://doi.org/10.1037/a0034733

de Sa Jr., A. R., Liebel, G., de Andrade, A. G., Andrade, L. H., Gorenstein, C., \& Wang, Y.-P. (2019). Can Gender and Age Impact on Response Patter of Depressive Symptoms among College Students? A Differential Item Functioning Analysis. Frontiers in Psychiatry, 10, 50. https://doi.org/10.3389/fpsyt.2019.00050

Essau, C. A., Lewinsohn, P. M., Seeley, J. R., \& Sasagawa, S. (2010). Gender Differences in the Developmental Course of Depression. Journal of Affective Disorders, 127, 185-190. https://doi.org/10.1016/j.jad.2010.05.016

Flett, G. L., Vredenburg, K., \& Krames, L. (1997). The Continuity of Depression in Clinical and Nonclinical Samples. Psychological Bulletin, 121, 395-416.

https://doi.org/10.1037/0033-2909.121.3.395

Frydrich, T., Dowdall, D., \& Chambless, D. L. (1992). Reliability and Validity of the Beck Anxiety Inventory. Journal of Anxiety Disorders, 6, 55-61. https://doi.org/10.1016/0887-6185(92)90026-4

Garland, E. L., Fredrickson, B., Kring, A. M., Johnson, D. P., Meyer, P. S., \& Penn, D. L. (2010). Upward Spirals of Positive Emotions Counter Downward Spirals of Negatively: Insights from the Broaden-and-Build Theory and Affective Neuroscience on the Treatment of Emotion Dysfunctions and Deficits in Psychopathology. Clinical Psychology Review, 30, 849-864. https://doi.org/10.1016/j.cpr.2010.03.002

Hale, W. W., Raaijmakers, Q., Muris, P., van Hoof, A., \& Mees, W. (2008). Developmental Trajectories of Adolescent Anxiety Disorder Symptoms: A 5-Year Prospective Community Study. Journal of the American Academy of Child \& Adolescent Psychiatry, 47, 556-564. https://doi.org/10.1097/CHI.0b013e3181676583

Hankin, B. J. (2009). Development of Sex Differences in Depressive and Co-Occurring Anxious Symptoms during Adolescence: Descriptive Trajectories and Potential Explanations in a Multiwave Prospective Study. Journal of Clinical Child \& Adolescent Psychology, 38, 460-472. https://doi.org/10.1080/15374410902976288

Hettema, J. M., Prescott, C. A., \& Kendler, K. S. (2003). The Effects of Anxiety, Substance Use, and Conduct Disorders on Risk of Major Depressive Disorder. Psychological Medicine, 33, 1423-1432. https://doi.org/10.1017/S0033291703008365

Hubley, S., \& Dimidjian, S. (2017). Behavior Therapy of Depression. In R. J. DeRubeis, \& D. R. Strunk (Eds.), The Oxford Handbook of Mood Disorders (pp. 423-434). Oxford: Oxford University Press. https://doi.org/10.1093/oxfordhb/9780199973965.013.36

Hutt, C. (1978). Biological Bases of Psychological Sex Differences. American Journal of Diseases of Children, 132, 170-177. https://doi.org/10.1001/archpedi.1978.02120270068015 
Jalnapurkar, I., Allen, M., \& Pigott, T. (2018). Sex Differences in Anxiety Disorders: A Review. Journal of Psychiatry, Depression, and Anxiety, 4, 1-9. https://doi.org/10.24966/PDA-0150/100011

Johnson, D. P., \& Whisman, M. A. (2013). Gender Differences in Rumination: A Meta-Analysis. Personality and Individual Differences, 55, 367-374. https://doi.org/10.1016/j.paid.2013.03.019

Julian, L. J. (2011). Measures of Anxiety: State-Trait Anxiety Inventory (STAO), Beck Anxiety Inventory (BAI), and Hospital Anxiety and Depression Scale-Anxiety (HADS-A). Arthritis Care and Research, 63, 467-472. https://doi.org/10.1002/acr.20561

Kendall, P. C., \& Brady, E. U. (1995). Comorbidity in the Anxiety Disorders of Childhood. In K. Craig, \& K. Dobson (Eds.), Anxiety and Depression in Adults and Children (pp. 3-6). Thousand Oaks, CA: Sage.

Kessler, R. C. (2007). The Global Burden of Anxiety and Mood Disorders: Putting ESEMeD Findings into Perspective. Journal of Clinical Psychiatry, 68, 10-19.

Kessler, R. C., Andrade, L. H., Bijl, R. V., Offord, D. R., Demler, O. V., \& Stein, D. J. (2002). The Effects of Co-Morbidity on the Onset and Persistence of Generalized Anxiety Disorder in ICPE Surveys. Psychological Medicine, 32, 1213-1225. https://doi.org/10.1017/S0033291702006104

Kessler, R. C., Berglund, P., Demler, O., Jin, R., Koretz, D., Merikangas, K. R., Rush, A. J., Walters, E. E., \& Wang, P. S. (2003). The Epidemiology of Major Depressive Disorder: Results from the National Comorbidity Survey Replication (NCS-R). Journal of the American Medical Association, 289, 3095-3105.

https://doi.org/10.1001/jama.289.23.3095

Kessler, R. C., Gruber, M., Hettema, J. M., Hwang, I., Sampson, N., \& Yonkers, K. A. (2008). Co-Morbid Major Depression and Generalized Anxiety Disorders in the National Comorbidity Survey Follow-Up. Psychological Medicine, 38, 365-374.

https://doi.org/10.1017/S0033291707002012

Kessler, R. C., Nelson, C. B., McGonagle, K. A., Liu., J., Swartz, M., \& Blazer, D. G. (1996). Comorbidity of DSM-III-R Major Depressive Disorder in the General Population: Results from the US National Comorbidity Survey. British Journal of Psychiatry, 168, 17-30. https://doi.org/10.1192/S0007125000298371

Kreitler, S. (2018). The Meaning Profiles of Anxiety and Depression: Similarities and Differences in Two Age Groups. Cognition and Emotion, 32, 1499-1513. https://doi.org/10.1080/02699931.2017.1311248

Lim, G. Y., Tam, W. W., Lu, Y., Ho, C. S., Zhang, M. W., \& Ho, R. C. (2018). Prevalence of Depression in the Community from 30 Countries between 1994 and 2014. Scientific Reports, 8, Article No. 2861. https://doi.org/10.1038/s41598-018-21243-x

Little, T. D. (2013). Longitudinal Structural Equation Modelling. New York: Guilford Press.

Little, T. D., Cunningham, W. A., Shahar, G., \& Widaman, K. F. (2002). To Parcel or Not to Parcel: Exploring the Question, Weighing the Merits. Structural Equation Modeling, 9, 151-173. https://doi.org/10.1207/S15328007SEM0902_1

Lovibond, P. F. (1998). Long-Term Stability of Depression, Anxiety, and Stress Syndromes. Journal of Abnormal Psychology, 107, 520-526. https://doi.org/10.1037/0021-843X.107.3.520

Moffitt, T. E., Harrington, H., Caspi, A., Kim-Cohen, J., Goldberg, D., Gregory, A. M., \& Poulton, R. (2007). Depression and Generalised Anxiety Disorder: Cumulative and Sequential Comorbidity in a Birth Cohort Followed Prospectively to Age 32 Years. 
Archives of General Psychiatry, 64, 651-660. https://doi.org/10.1001/archpsyc.64.6.651

Nolen-Hoeksema, S., \& Jackson, B. (2001). Mediators of the Gender Difference in Rumination. Psychology of Women Quarterly, 25, 37-47. https://doi.org/10.1111/1471-6402.00005

Osman, A., Downs, W. R., Barrios, F. X., Kopper, B. A., Gutierrez, P. M., \& Chiros, C. E. (1997). Factor Structure and Psychometric Characteristics of the Beck Depression Inventory-II. Journal of Psychopathology and Behavioral Assessment, 19, 359-376. https://doi.org/10.1007/BF02229026

Parker, G., \& Hadzi-Pavlovic, D. (2004). Is the Female Preponderance in Major Depression Secondary to a Gender Difference in Specific Anxiety Disorders? Psychological Medicine, 34, 461-470. https://doi.org/10.1017/S0033291703001181

Ramakrishna, S., Cooklin, A. R., \& Leach, L. S. (2019). Comorbid Anxiety and Depression: A Community-Based Study Examining Symptomology and Correlates during the Postpartum Period. Journal of Reproductive and Infant Psychology, 37, 468-479. https://doi.org/10.1080/02646838.2019.1578870

Rose, A. J. (2002). Co-Rumination in the Friendships of Girls and Boys. Child Development, 73, 1830-1843. https://doi.org/10.1111/1467-8624.00509

Salk, R. H., Hyde, J. S., \& Abramson, L. Y. (2017). Gender Differences in Depression in Representative National Samples: Meta-Analyses of Diagnoses and Symptoms. Psychological Bulletin, 143, 783-822. https://doi.org/10.1037/bul0000102

Skouteris, H., Wertheim, E. H., Rallis, S., Milgrom, J., \& Paxton, S. J. (2009). Depression and Anxiety through Pregnancy and the Early Postpartum: An Examination of Prospective Relationships. Journal of Affective Disorders, 113, 303-308. https://doi.org/10.1016/j.jad.2008.06.002

Starr, L. R., Stroud, C. B., \& Li, Y. I. (2016). Predicting the Transition from Anxiety to Depressive Symptoms in Early Adolescence: Negative Anxiety Response Style as a Moderator of Sequential Comorbidity. Journal of Affective Disorders, 190, 757-763. https://doi.org/10.1016/j.jad.2015.10.065

Tabachnick, B. G., \& Fidell, L. S. (2019). Using Multivariate Statistics (7th ed.). New York: Pearson Publishers.

Vredenburg, K., Flett, G. L., \& Krames, L. (1993). Analogue versus Clinical Depression: A Critical Reappraisal. Psychological Bulletin, 113, 327-344. https://doi.org/10.1037/0033-2909.113.2.327

Watson, D., \& Kendall, P.C. (1989). Understanding Anxiety and Depression: Distinctive and Overlapping Features. In P. C. Kendall, \& D. Watson (Eds.), Personality, Psychopathology, and Psychotherapy (pp. 3-26). San Diego, CA: Academic Press, Inc.

Watson, D., Clark, L. A., Weber, K., Assenheimer, J. S., Strauss, M. E., \& McCormick, R. A. (1995). Testing a Tripartite Model: II. Exploring the Symptom Structure of Anxiety and Depression in Student, Adult, and Patient Samples. Journal of Abnormal Psychology, 104, 15-25. https://doi.org/10.1037/0021-843X.104.1.15

Whisman, M. A., \& Richardson, E. D. (2015). Normative Data on the Beck Depression Inventory-Second Edition (BDI-II) in College Students. Journal of Clinical Psychology, 71, 898-907. https://doi.org/10.1002/jclp.22188

Wittchen, H. U., Kessler, R. C., Pfister, H., \& Lieb, M. (2000). Why Do People with Anxiety Disorders Become Depressed? A Prospective-Longitudinal Community Study. Acta Psychiatrica Scandinavica, 102, 14-23. https://doi.org/10.1111/j.0065-1591.2000.acp29-03.x

Wood, D., Crapnell, T., Lau, L., Bennett, A., Lotstein, D., Ferris, M., \& Kuo, A. (2018). 
Emerging Adulthood as a Critical Stage in the Life Course. In N. Halfon, C. Forrest, R. Lerner, \& E. Faustman (Eds.), Handbook of Life Course Health Development (pp. 123-143). New York: Springer. https://doi.org/10.1007/978-3-319-47143-3_7 